\title{
Targeting Adult Neurogenesis to Optimize Hippocampal Circuits in Aging
}

\author{
Kathleen M. McAvoy ${ }^{1,2,3}$ • Amar Sahay ${ }^{1,2,3,4}$
}

Published online: 23 May 2017

(C) The American Society for Experimental NeuroTherapeutics, Inc. 2017

\begin{abstract}
Millions of individuals suffer from age-related cognitive decline, defined by impaired memory precision. Increased understanding of hippocampal circuit mechanisms underlying memory formation suggests a role for computational processes such as pattern separation and pattern completion in memory precision. We describe evidence implicating the dentate gyrus-CA3 circuit in pattern separation and completion, and examine alterations in dentate gyrus-CA3 circuit structure and function with aging. We discuss the role of adult hippocampal neurogenesis in memory precision in adulthood and aging, as well as the circuit mechanisms underlying the integration and encoding functions of adult-born dentate granule cells. We posit that understanding these circuit mechanisms will permit generation of circuit-based endophenotypes that will edify new therapeutic strategies to optimize hippocampal encoding during aging.
\end{abstract}

Keywords Aging $\cdot$ Neurogenesis $\cdot$ Dentate gyrus ·

Hippocampus $\cdot$ Memory $\cdot$ Pattern separation

Amar Sahay

asahay@mgh.harvard.edu

1 Center for Regenerative Medicine, Massachusetts General Hospital, Boston, MA 02114, USA

2 Harvard Stem Cell Institute, Cambridge, MA 02138, USA

3 Department of Psychiatry, Massachusetts General Hospital, Harvard Medical School, Boston, MA 02114, USA

4 BROAD Institute of Harvard and MIT, Cambridge, MA 02142, USA

\section{Introduction}

The world's population is aging, with $\sim 20 \%$ of the US population expected to be older than 65 years in 2030 (up from 13\% in 2010). Aging is associated with impairments across cognitive domains, including memory, attention, and executive function [1-4]. Cognitive decline affects a large portion of those $>65$, where $12 \%$ of individuals self-reported increased memory loss in the preceding year [5] and nearly $60 \%$ of elderly study subjects showed cognitive decline over an 8-year study [6]. Clinically, memory impairments are diagnosed by performance on a number of tests, as well as self- and family reports [7, 8]. Aged individuals show select impairments in tasks requiring encoding new memories of events, facts, or contextual and spatial information, whereas short-term memory, autobiographical memory, and semantic and procedural knowledge remain relatively stable (reviewed in [1,9]).

Aging also increases the incidence of pathological neurodegenerative diseases, including Alzheimer's disease and mild cognitive impairment (MCI), a clinical criteria used for early stage cognitive dysfunction preceding Alzheimer's disease. Similar memory impairments are seen in normal aging and MCI, although the deficits in MCI generally exceed those found in normally aging individuals [10]. Considerable evidence implicates dysfunction of the medial temporal lobe in age-associated memory impairments and MCI $[1,11,12]$. Notably, by examining aging across species that do not show neurodegenerative disease, age-related and disease-related impairments can be disentangled. Impaired memory has been described as a symptom of normal aging as it occurs across species [9]. Dissociating normal and pathological aging allows for the testing of empirical models to determine the neuroanatomical and functional basis of behavioral impairments, and the efficacy of age- or disease-specific treatments. 
The identification of new therapeutics for age-associated memory impairments necessitates identification of underlying core neurobiological changes, both cellular and circuit-based. Hippocampal circuits support declarative or explicit memory function, and in humans this type of memory includes memories with a what-when-where component, including personal experiences and events that can be recalled and described, as well as spatial memory. In humans, our ability to assess age-related hippocampal changes is limited to behavioral measures, imaging analysis [including magnetic resonance imaging (MRI)/functional MRI (fMRI)], and postmortem histological assessment, whereas animal models with homologous hippocampal functions [13, 14], permit greater ability to interrogate the causal relationships between circuitry and behavior.

Here, we will present the case that disruption of dentate gyrus (DG)- and CA3-dependent operations such as pattern separation and completion, which are thought to underlie episodic memory formation, contribute to age-associated memory impairments. We describe evidence implicating the DGCA3 circuit in pattern separation and completion and examine alterations in DG-CA3 circuit structure and function with aging. We discuss the role of adult hippocampal neurogenesis in memory precision in adulthood and aging, as well as the circuit mechanisms underlying the integration and functions of adult-born dentate granule cells (DGCs). We posit that understanding these circuit mechanisms will edify new circuit based strategies to optimize hippocampal encoding in aged individuals. Furthermore, such efforts will permit generation of circuit-based endophenotypes that facilitate objective monitoring of symptom progression and treatment efficacy.

\section{Memory Precision is Impaired With Aging}

Episodic memory is particularly vulnerable to the deleterious effects of age. Older adults show deficits relative to young adults on tasks that require the formation of new episodic memories, including those with spatial and contextual components (reviewed in $[1,13,15])$. The literature on age-related memory impairments across species has been reviewed in detail elsewhere [13, 14], and data indicate that these memory deficits cannot be attributed to deficits in sensory, motor, or motivational deficits [16-18].

While many facets of memory are impaired with aging, growing evidence supports significant alterations in behaviors that require distinguishing among experiences that share similar elements, or conditions that require resolution of memory interference [14]. With aging, key changes in episodic memories are: 1) loss of contextual details (e.g., spatial or visual detail about stimulus location [19]); 2) susceptibility to interference (e.g., difficulty in remembering target stimuli when similar items or "lures" are used [20,21]); and 3) a decreased response to new stimuli (e.g., impairments in identifying whether an item is new [22-24]; reviewed in [14, 25]).
These deficits lead to specific memory errors, such as "false recognition" and a phenotype of "memory rigidity" [26]. It has been proposed that age-related deficits in memory precision may be due to alterations in neural mechanisms underlying memory formation and retrieval, such as pattern separation and completion (reviewed in $[14,25,26])$.

\section{The DG-CA3 Circuit Supports Pattern Separation and Pattern Completion in Rodents and Humans}

How does the hippocampus support creating new episodic memories that are distinct from similar, previously stored memories? Pattern separation is the computational process of making similar inputs less similar in output (i.e., orthogonalization) [27-29]. When inputs are very different, pattern separation is not required as the differences in input are sufficient to generate divergent outputs. However, when inputs are very similar (and thus likely to generate similar outputs) a computational process to generate distinct outputs is required to disambiguate these similar inputs. For memory encoding, this process is hypothesized to include encoding of new memories as nonoverlapping neuronal ensembles. Complementing the process of pattern separation is the process of pattern completion, defined as a network's ability to recall a complete representation when presented with incomplete or degraded input. While pattern separation and pattern completion are complementary processes, the DG performs pattern separation, and CA3 performs pattern separation or completion [30-33] (reviewed in [34, 35]). Recordings from DG and CA3 suggest that the DG automatically orthogonalizes inputs, and that it is the autoassociative network in CA3 that determines whether these inputs will be stored as a new memory pattern or if an old memory will be retrieved $[35,36]$. Therefore, generating sufficiently orthogonalized information in DG and exporting this to CA3 is critical to avoid inappropriate pattern completion and incorrect memory retrieval.

Pioneering behavioral studies uncovered a role for the DGCA3 circuit in resolution of memory interference (reviewed in [36]). Rats with selective lesions of the CA3 region were impaired in spatial discrimination of objects closely or widely spaced apart [37], whereas DG lesions produced impairments only at small separations, and CA1 lesions did not affect discrimination [38]. DG-specific $N$-methyl-D-aspartate receptor 1 deletion impaired discrimination of similar contexts [39], whereas CA3-specific deletion of NR1 revealed that CA3 is critical for memory retrieval when inputs are degraded [40]. These findings are consistent with the hypothesized role for the DG in pattern separation [27,35] and with the postulated role for CA3 in pattern completion, which was based on its recurrent collaterals allowing the region to act as an autoassociative network (Fig. 1) [41-43].

Evidence for pattern separation requires demonstration of transformation of inputs into more divergent outputs at a 
A
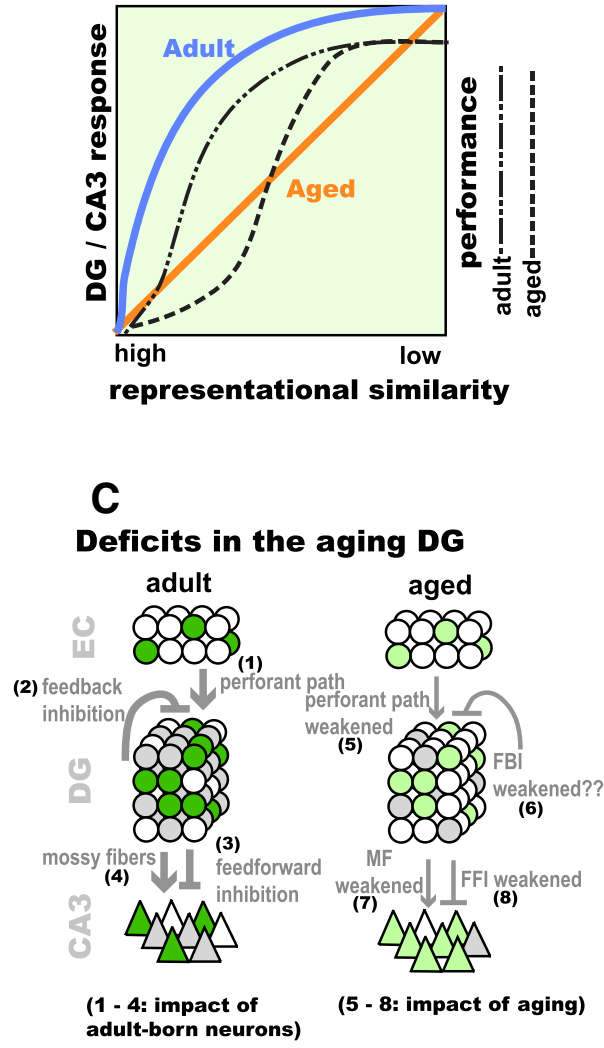

Fig. 1 Dentate gyrus (DG)-CA3 circuitry and pattern separation. (a) Schematic description of DG-CA3 blood oxygen level-dependent responses (left axis, blue and orange lines) and discrimination accuracy (right axis, dashed lines) in adults and aged individuals in a task in which image stimuli were presented and were varied on a continuum from repeat presentation, to similar items or lures, to novel foils (adapted from [14, 26], based on data presented in [23, 24, 72]). Aged individuals showed a failure to activate the DG-CA3 region when similar items or lures were presented. When explicitly tasked with identifying the images as novel or familiar, aged individuals performed more poorly when the images were most similar. (b) The DG performs pattern separation on incoming inputs from the entorhinal cortex (EC). The large number of DGCs relative to the EC ("input expansion") and the sparse activation of the DG are thought to facilitate the orthogonalization of similar inputs into distinct
B

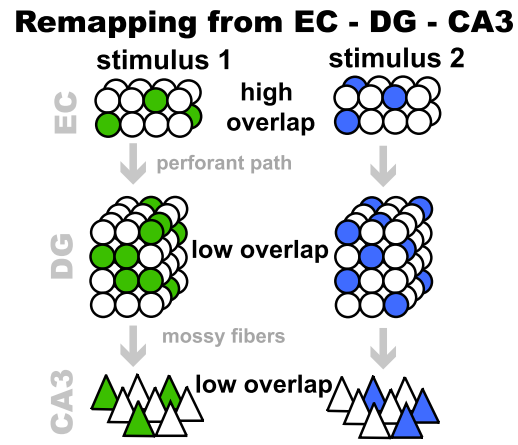

D

\section{Adult-born GCs modify encoding}

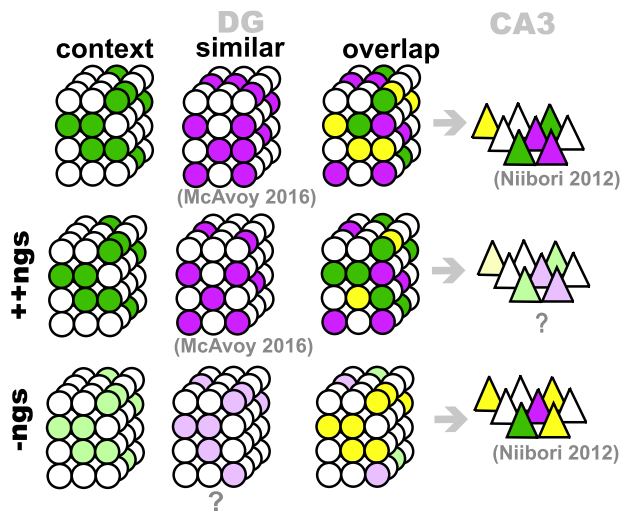

outputs. (c) The DG is modified by both incorporation of adult-born DGCs and by aging. 1) Adult-born DGCs may be more responsive to weak perforant path inputs. 2) Adult-born DGCs recruit feedback inhibition (FBI) onto the DG, and (3) feed-forward inhibition (FFI) onto CA3. 4) Adult-born DGCs exhibit increased plasticity at the DGCA3 synapse. 5) In aged animals, perforant path inputs to DG are weaker, and feedback inhibition (6), and feedforward inhibition (8), may be decreased. 7) CA3 exhibites higher basal activity and hyperexcitability. (d) Adult-born DGCs promote population- based coding in DG and CA3. Enhancing adult hippocampal neurogenesis (ngs) decreases overlap between DG ensembles encoding similar contexts [118]. Decreasing adult hippocampal neurogenesis increases overlap between CA3 ensembles encoding similar contexts [146] neural level. Pattern separation in the DG is supported by encoding of similar inputs by differences in firing rates of place cells (rate remapping) or recruitment of nonoverlapping ensembles of neurons [global remapping (Fig. 1); [33, 35, 44, 45]]. Knierim et al. [35] performed simultaneous recordings from entorhinal cortex (EC) and DG, and DG and CA3, as rats explored an environment in which local and distal environmental cues were gradually morphed. They showed that the DG remaps in response to small changes in context, under conditions where remapping in $\mathrm{EC}$ and $\mathrm{CA} 3$ remained unchanged $[35,44,46]$. A previous study had found that the DG also undergoes rate remapping for subtle changes in environment under conditions in which EC grid cell activity was unchanged [33]. Interestingly, the active cells with multiple place fields recorded in this study have been suggested to be hilar mossy cells and not DGCs [47-50]. Thus, DGCs and mossy cells may both contribute to pattern separation in the DG through different mechanisms.

Human studies have demonstrated that the DG-CA3 circuit is preferentially engaged under conditions of high memory interference [51-54]. Human imaging studies have taken advantage of the fact that repetition of the same stimuli suppresses blood oxygen level-dependent responses upon subsequent presentation (the repetition-suppression effect), and tested individuals with presentation of the same visual stimuli, novel stimuli (or "foils"), or similar stimuli ("lures") while the 
subjects were engaged in an unrelated labeling task (e.g., categorizing objects as outdoors or indoors). Equivalent activation in response to targets and similar lures suggests hippocampal engagement for discrimination of the lures as novel stimuli, whereas the repetition-suppression effect indicates that the stimuli were perceived as repeat presentations. In contrast to CA1 and EC, similar levels of DG/CA3 activation in response to previously seen stimuli and lures was observed, indicating preferential engagement of DG/CA3 to discrimination of similar stimuli $[51,53]$. Advances in imaging technology that overcome limitations in resolution will enable determination of input-output EC-DG transformations during these tasks.

Interestingly, a new study describes a patient, BL, with bilateral DG damage [55]. When given a visual recognition task similar to that described above, BL showed similar performance to controls when identifying novel and similar stimuli (lures), indicating intact recognition memory, but was 5 times more likely than controls to identify lures as previously seen stimuli. This indicates a deficit in resolution of interference, and was interpreted by the authors as a failure of pattern separation. However, in a second test that evaluates recognition memory performance with 10 drawings of rooms, 5 of which were familiar and 5 of which were novel, presented unmasked or masked to varying degrees, $\mathrm{BL}$ also showed a strong tendency to identify incomplete scenes as familiar, even when they were novel. The authors interpreted his poor performance identifying the unmasked images as indicative of a failure of pattern separation, as the image similarity presumably generated memory interference. Further, the authors note that patient BL's significantly worse performance on the degraded inputs (masked incomplete images) suggests a further bias toward pattern completion. These observations are consistent with the hypothesis that orthogonalized inputs from DG to CA3 are necessary to prevent CA3's attractor network from reactivating a previously stored memory pattern $[35,36]$.

\section{Age-Related Changes in DG-CA3 Functions in Rodents}

Aged rats demonstrate selective deficits in tasks requiring resolution of memory interference. Using a spatial memory test in which the animal had to distinguish a correct, learned reward arm from either a nonadjacent or adjacent nonreward "bait" arm, Gracian et al. [56] found that aged rats showed a particular impairment (relative to adult rats) when the nonreward "bait" arm was adjacent to the correct reward arm.

Deficits in behavioral memory precision may be due to failure to reactivate and remap neuronal ensembles. Experimental data in rodents substantiates the hypothesis that ensembles of cells encode information about spatial contexts. Reactivation of unique ensembles of DGCs previously activated (expressing the immediate early gene, $c f o s$ ) by a particular context is sufficient to trigger context-specific behavior
$[57,58]$. In rats, $\mathrm{CA} 3$ and $\mathrm{CA} 1$ pyramidal neurons and DGCs fire during traversal of a limited portion of an environment (the "place field" of the cell; e.g. [59]). By reconstructing the firing patterns of many simultaneously recorded cells (the place cell map), the animal's location within the environment can be predicted (e.g. [60]). These maps are modified by experience-dependent changes in synaptic efficacy to allow for association of specific memory features (reviewed in [61]).

Aged rats fail to retrieve the same place cell "map" in CA1 - retrieving the incorrect place field sequence for a second visit to an environment more often than do young rats with equivalent experience [62]. Further, aged rats failed to realign place fields to room cues, a feature that correlated with poor learning of a goal location [63]. Aged rats also showed reduced changes to $\mathrm{CA} 3 / \mathrm{CA} 1$ place fields with alteration of the environment $[64,65]$, and showed reduced remapping in an entirely new context $[65,66]$; CA3 cells, in particular, showed this "representational rigidity or inflexible remapping" and higher firing rates, in general [67]. To reconcile reports of incorrect map retrieval [62] and representational rigidity [64-67], Wilson et al. [68] recorded CA3/CA1 place cells during sequential exposures to a novel environment. They found that when aged rats were exposed to a new environment, CA1/CA3 place cell firing was initially unchanged, requiring greater environmental change to remap, followed by a period where the place cells became linked to the external cues (delayed relative to adult rats; see also [63]), and, finally, followed by a period where place cell ensembles showed incorrect map retrieval, analogous to that described by Barnes et al. [62] (reviewed in [9, 26]).

Using the catFISH technique (cellular compartment analysis of temporal activation using fluorescent in situ hybridization [69]) to compare ensemble overlap in the adult and aged DG upon re-exposure to the same context or upon exposure to a different context, Marrone et al. [70] found that there is less overlap in activation of DGCs upon re-exposure to the same context, indicating a failure to retrieve the appropriate map, reminiscent of CA3 place cell activity patterns in aged rats $[62,68,70]$. Importantly, this failure was limited to reexposure to the same context, and was not seen with reexposure to a different context in a different room. In a second task, rats explored an identical Y-maze in 2 different rooms sequentially, and were tasked with visiting a different novel arm in each room. Aged rats showed poorer performance relative to adults, which correlated with failure to reactivate a similar ensemble [70]. Thus, impaired ensemble reactivation in DG, as in CA3 (reviewed in [26]), in aged rats correlates with poor spatial memory precision.

In sum, data from aged rats suggest that CA3 fails to appropriately remap to novelty, and instead incorrectly retrieves previous memory patterns. Although technical difficulties have limited our ability to interrogate age-related changes in place field maps or in pattern separation in the DG, 
considerable evidence implicates structural alterations in DG and CA3 that may underlie inefficiencies in ensemble reactivation and remapping in aging.

\section{Age-Related Changes in DG-CA3 Functions in Humans}

Aged individuals show specific deficits on tasks that engage the DG-CA3 circuit. In a test evaluating recognition memory performance with identification of complete or incomplete novel or familiar room drawings, aged individuals showed poor performance on discrimination of novel versus familiar stimuli, a phenotype construed by authors to reflect impaired pattern separation [71]. Further, aged individuals incorrectly identified degraded, novel images as familiar, suggesting a bias towards pattern completion, reminiscent of patient $\mathrm{BL}$ $[55,71]$. In another visual recognition test in which individuals were presented with repeats of the same visual stimuli, novel "foils", or similar "lures", older adults showed poor discrimination accuracy when stimuli and lures were very similar, and exhibited comparable performance as adults only in discerning distinct stimuli [23, 24]. Interestingly, aged individuals exhibited increased blood oxygen level-dependent signal in DG/CA3 in response to both original or target stimuli, as well as lures [24]. Older adults also required more dissimilarity in stimuli and lures to decrease the DG/CA3 repetition-suppression effect $[24,25,72]$. Interestingly, individuals with MCI exhibited impaired discrimination of lures and elevated DG-CA3 activity during presentation of lures [73]. Newer approaches using ultra-high-resolution fMRI at $7 \mathrm{~T}$ and multivariate pattern analysis will permit assessment of DG and CA3 activation separately (rather than as DG-CA3) in aged individuals and individuals with MCI using these tasks [53].

\section{Age-Related Changes in DG-CA3 Structure and Connectivity}

The aged hippocampus does not show loss of principal cells in humans [74], rats [75, 76], monkeys [77], or mice [78] (reviewed in [9]), suggesting that age-associated memory impairment is more likely due to synapse loss and dysfunction. Consistent with this notion, the hippocampus is reduced in size in aged humans [79-81] (reviewed in [82]), and size correlates with deficits in explicit memory in aged individuals [83, 84]. Within the hippocampus, functional imaging studies suggest that the DG is the region most profoundly affected by aging in rats and macaques [85] (reviewed in [82]). In rodents, there is decreased EC to DG synapse number [86, 87] (reviewed in [88]) and decreased perforant path presynaptic fiber potential amplitudes [89]. Furthermore, long-term potentiation at the perforant path/DG synapse is more difficult to induce and shows reduced persistence in aged rats [90] (reviewed in [91, 92]). Concomitant with these EC-to-DG changes, there is a reduction in activity as measured by immediate early gene induction in DG following behavior in aged rats $[70,85,93]$. Diffusion-tensor imaging suggests a reduction in perforant path connectivity from EC to DG in aged humans [24, 72, 94-96]. Further, this reduction correlated with representational rigidity and poor behavioral discrimination in aged humans [72], further supporting the idea that changes in $\mathrm{EC}$ to DG connections drive aged-related impairments.

In contrast to decreased DG activation [70, 85, 93], CA3 shows hyperexcitability and elevated firing rates in rodents and nonhuman primates [67, 97-99]. Because mossy fibers of DGCs synapse onto inhibitory interneurons and CA3 pyramidal neurons $[100,101]$, decreased perforant path inputs onto DG (reviewed in $[88,92]$ ) may reduce feed-forward inhibition from the DG to CA3. In addition, impaired plasticity at mossy fiberinterneuron synapses (reviewed in [102]) may underlie hyperactivity in CA3 [98]. Whole-cell recordings in CA3 indicate that the increased intrinsic excitability of aged pyramidal neurons is accompanied by a reduction in feed-forward inhibition onto CA3 [98]. Further, the number of inhibitory interneurons, particularly in the hilus, is reduced with age [103-105]. These findings suggest altered EC-DG connectivity and feed-forward inhibition from DG to CA3 may contribute to CA3 hyperactivation.

\section{Adult Hippocampal Neurogenesis Decreases With Aging and Adult-Born DGCs Contribute to Pattern Separation}

New DGCs are generated from neural stem cells in the subgranular zone of the DG throughout life (Fig. 2). Pioneering work by Altman and Das (1965) [106] first identified adult neurogenesis in the subgranular zone of the DG in rodents, and more recent studies have confirmed that the DG supports adult hippocampal neurogenesis in humans [107-111]. Adult hippocampal neurogenesis is exquisitely sensitive to circuit demands, and is tightly regulated by discrete, yet overlapping, mechanisms at multiple stages of neuronal maturation to ensure appropriate additions to the circuit (reviewed in [112-114]). In the subgranular zone of the adult DG, active neural stem cells give rise to transiently active neural progenitors, which differentiate into immature DGCs. Immature DGCs receive afferent connections in a stereotyped order [115-118], and the acquisition of these inputs is thought to govern their survival and integration into the hippocampus (Fig. 2) [118-122]. Adult-born DGCs between $\sim 4$ and 8 weeks of age exhibit heightened plasticity and are hypothesized to preferentially contribute to memory processing [118, 123-132].

Although adult neurogenesis declines slowly with age in humans $[110,111]$, it declines precipitously in mice, with $50 \%$ to $95 \%$ reduction between 6 weeks and 5 months (see, e.g. [118, $133,134]$; reviewed in $[135,136])$. As this timeline is much faster than the acquisition of age-related memory impairments, it does not appear that declines in neurogenesis drives many aspects of cognitive decline [88, 137]. Nevertheless, if neurogenesis is important for encoding, then stimulation of 


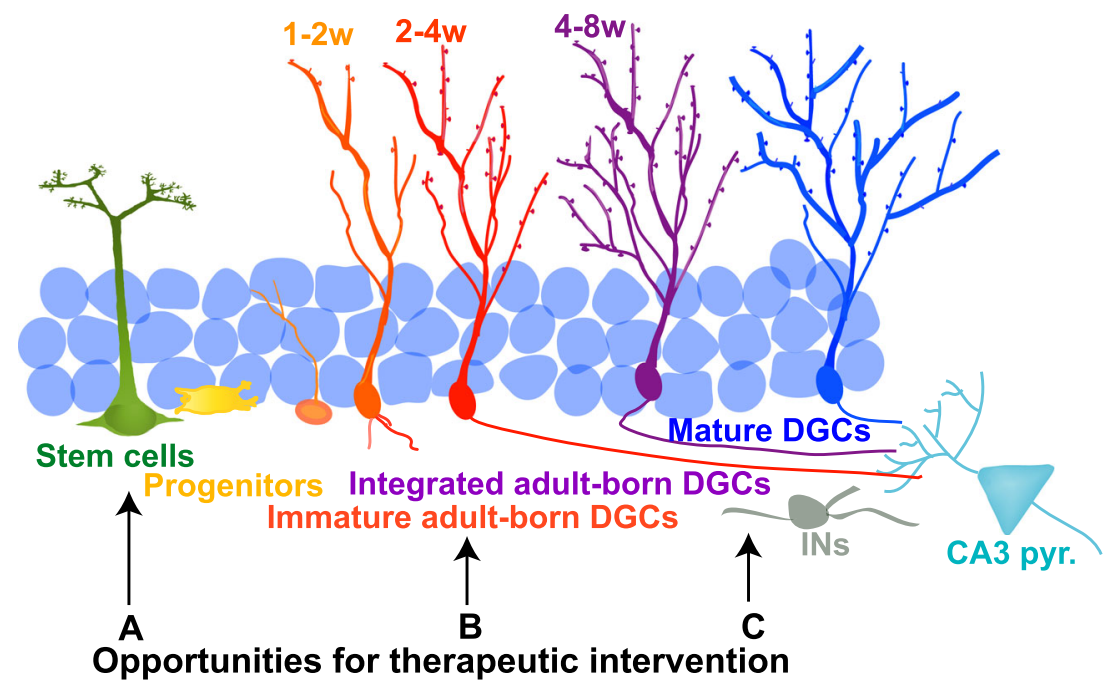

Fig. 2 Schematic of adult hippocampal neurogenesis and opportunities for intervention. Adult-born dentate granule cells (DGCs) arise from neural stem cells in the subgranule zone of the DG, with activated neural stem cells giving rise to transiently active progenitors, which differentiate into immature DGCs. Immature DGCs form afferent and efferent connections in a stereotyped order, and the acquisition of these inputs (initially $\gamma$-aminobutyric acid, and then glutamatergic inputs from the entorhinal cortex) governs their survival and integration into the hippocampus. Opportunities for therapeutic intervention to increase the adult-born DGC population or to mimic their effects on the circuit are highlighted: (a) removal of age-related molecular breaks on stem-cell activation and proliferation; (b) promoting integration of adult-born DGCs; (c) modulating DGC connectivity to increase feed-forward and feedback inhibition. IN Interneuron neurogenesis may represent a therapeutic strategy to improve encoding in aging.

Ablation of adult-born DGCs impairs performance in behavioral tasks that require resolution of memory interference [138-146] (reviewed in [147]). Abrogation or reduction of adult hippocampal neurogenesis, or decreasing survival of adult-born DGCs, impaired discrimination of similar contexts [139, 141, $142,148]$. In a complementary series of experiments, deleting the proapoptotic gene Bax from adult-born DGCs, and increasing their survival, resulted in improved contextual discrimination [141]. Consistent with this finding, genetic silencing of the output of the majority of mature DGCs (4-5 weeks of age and older) resulted in improved contextual discrimination [139], suggesting that adult-born DGCs are sufficient for this discrimination. Interestingly, these mice were impaired in pre-exposuredependent contextual fear conditioning and in using partial spatial cues to find a hidden platform in the water maze. These data suggest that mature DGCs may contribute preferentially to recall of memories based on partial cues, potentially through pattern completion [139].

Optogenetic silencing of adult-born DGCs during memory retrieval, or training in the contextual fear discrimination learning task, resulted in memory impairments [126, 149]. Interestingly, silencing adult-born DGCs during re-exposure to the trained, shock context had no effect on freezing, but silencing adult-born DGCs during exposure to the novel "safe" context prevented discrimination [149]. This suggests that adult-born DGCs may be required during encoding of a novel context.
Additional evidence for a role for adult-born DGCs in resolving memory interference comes from tasks using the radial arm maze and Morris water maze (MWM). Clelland et al. [138] showed that irradiation-dependent blockade of adult hippocampal neurogenesis produced deficits in both a radial arm maze task, in which mice had to chose a nonvisited arm from 1 of 2 options separated by varying degrees, and a touchscreen task in which choices varied by different spatial separations. In a complementary experiment, running, a proneurogenic intervention that also has other pleiotropic effects on circuitry, promoted performance on the touchscreen task [150]. Studies using the MWM have found that ablation of adult-born DGCs impairs spatial memory only when the target location of the hidden platform is reversed [143, 144]. In this reversal-learning portion, there is increased interference from the original location. Similarly, using a rotating platform in which a shock was given only in a particular quadrant, Burghardt et al. [145] found that ablation of adult-born DGCs impaired learning when the shock location was reversed. More recently, we engineered an inducible and reversible genetic system to modulate neuronal competition in the DG and enhance integration of 5 to 8-week-old adult-born DGCs [118]. In the MWM paradigm, increasing the population of 5 to 8-week-old adult-born DGCs had no effect on acquisition of initial platform location, but improved performance on the reversal portion of the task [118]. A direct, acute role for adult-born DGCs in resolution of memory interference comes from a study showing that optogenetic silencing of adult-born DGCs impaired a location discrimination task only 
if the adult-born DGCs matured during the acquisition phase of the task [132]. Thus, in rodents, substantial evidence supports a role for adult-born DGCs in behaviors requiring resolution of interference, raising the question as to what are the neural mechanisms by which adult-born DGCs contribute to DG-CA3 circuit functions.

\section{Circuit Mechanisms Underlying the Functions of Adult-Born DGCs in Memory Encoding}

\section{Adult-Born DGCs Exert Feedback Inhibition Onto the DG to Promote Sparseness}

Adult-born DGCs may modify the activity of the entire DG by recruiting feedback inhibition that affects the mature DGC population (Fig. 1) [151-156]. Within the DG, activation of local interneurons is critical to limit the size of the neuronal ensemble [157]. Several converging lines of evidence suggest that adult-born DGCs recruit feedback inhibition. Lacefield et al. [158] found that the synchrony of gamma oscillations were increased in the DG of mice in which neurogenesis was ablated, suggesting that local inhibitory microcircuits are altered with ablation of adult-born DGCs. Using a genetic model to increase neurogenesis in combination with voltagesensitive dye imaging in slices, we showed that increasing the adult-born DGC population decreased activity of the DG and increased threshold of activation of mature DGCs [152]. More recently, it was shown using channelrhodopsin stimulation of adult-born DGCs and whole-cell recordings that activation of the adult-born DGC population resulted in increased feedback inhibition onto mature DGCs $[155,159]$. Interestingly, feedback inhibition was driven more strongly by 6-week-old than 4-week-old adult-born DGCs [159]. Further, middle-aged mice showed a dramatic decrease in feedback inhibition [155]. Using similar techniques, Restivo et al. [160] showed that activation of 6 to 8-week-old DGCs was correlated with activation of DG interneurons.

A recent study suggested that adult-born DGCs may modulate excitatory synaptic transmission onto mature DGCs (and, consequently, sparseness) through neuronal competition and redistribution of perforant path-DGC synapses. The authors found that genetic expansion of the adult-born DGC population by conditional deletion of the proapoptotic gene Bax in adult neural stem cells reduced excitatory postsynaptic currents (EPSCs) in mature DGCs. Conversely, genetically ablating adult-born DGCs increased EPSCs in mature DGCs [161].

Analyses of DG activity in awake behaving animals following manipulations of adult hippocampal neurogenesis has suggested a role for adult-born DGCs in modulating sparseness in a mismatch-dependent manner. Burghardt et al. [145] found that ablation of adult-born DGCs decreased sparseness only under conditions of high interference (reversal learning on a rotating shock task). In vivo recordings in the DG during this task revealed a reversal learning-induced suppression of DGC responses that was lost following ablation of adult hippocampal neurogenesis [162]. Recently, we showed using cfos catFISH that genetically enhancing adult hippocampal neurogenesis decreased overlap between cellular ensembles activated in response to exposure to 2 similar (high interference), but not same or distinct (low interference), contexts. Further, we found that mice with increased adult hippocampal neurogenesis exhibited a mismatch-dependent suppression of activity in the DG [118]. Together, these observations demonstrate a role for adult-born DGCs in promoting sparseness and population- based coding mechanisms, such as global remapping, underlying pattern separation in the DG (Fig. 1) [118].

\section{Adult-Born DGCs May Modulate Feed-Forward Inhibition in DG-CA3 Circuit}

Learning induces structural changes of mossy fiber synapses onto CA3 stratum lucidum interneurons and this form of structural plasticity correlates with memory precision [163, 164]. Maturation of adult-born DGCs is accompanied by reduction in DGC-interneuron connectivity, as 4 to 6-week-old adultborn DGCs exhibit greater connectivity with CA3 stratum lucidum interneurons [160] (N. Guo and A. Sahay, unpublished observations). Although adult-born DGCs recruit feed-forward inhibition onto CA3 in vitro [159], the functional significance of DGC-interneuron connectivity and its relationship with ensemble dynamics and population-based coding mechanisms remains poorly understood.

Clues to how adult-born DGC-dependent recruitment of feed-forward inhibition in DG-CA3 or DGC-CA3 stratum lucidum interneuron connectivity affects encoding come from studies examining the impact of manipulating adult hippocampal neurogenesis on ensemble activation during memory retrieval. A study using genetic ensemble labeling tools found that ablating adult hippocampal neurogenesis (by irradiation) or decreasing adult hippocampal neurogenesis (by a behavioral stressor) decreased ensemble reactivation overlap in CA3 when mice were re-exposed to the training context several days following training [165], reminiscent of a failure to retrieve context-appropriate ensembles [26, 62]. These findings are consistent with previous studies showing at a behavioral level that blocking [166-169] or enhancing adult hippocampal neurogenesis [170] decreases (or increases, respectively) the strength of long-term memory. Recent work from our laboratory [118] showing that expansion of the population of 5 to 8week-old adult-born DGCs improved long-term contextual fear memory 4 weeks following training, lends further support to a role for adult-born DGCs in promoting long-term memory. 
Analysis of population-based coding in CA3 using catFISH has shed light on how adult hippocampal neurogenesis may support pattern separation in CA3. Pharmacological or genetic reduction of adult hippocampal neurogenesis in mice resulted in an increase in overlap of cellular ensembles in CA3 when mice were exposed to similar, but not distinct, contexts. Interestingly, this increase in overlap was driven by increased activation of CA3, or decreased sparseness, when mice were exposed to the second context (Fig. 1d) [146].

Thus, adult-born DGCs may recruit feed-forward inhibition to dictate ensemble dynamics underlying long-term memory and global remapping in CA3. Further studies will delineate circuit mechanisms by which adult-born DGCs affect CA3 ensemble dynamics and whether increasing adult hippocampal neurogenesis during aging is sufficient to reverse failures of appropriate ensemble activation and remapping [26, 70].

\section{Targeting Adult Neurogenesis to Improve DG-CA3 Functions in Aging}

\section{Promoting Integration of Adult-Born DGCs Into the Hippocampal Circuit}

Previous studies have suggested that that stimulating neurogenesis throughout life improves memory in aging [171-177] (reviewed in [178]), although the correlation between levels of adult neurogenesis in aged animals and memory performance is tenuous $[179,180]$. We sought to address the question of whether enhancing adult hippocampal neurogenesis in aged mice causally improves memory. To accomplish this, we developed a strategy to modulate neuronal competition and promote integration of adult-born DGCs without affecting olfactory bulb neurogenesis. Immature DGCs receive afferent connections in a stereotyped order [115-118], and the acquisition of these inputs is thought to govern their survival and integration into the hippocampus (Fig. 2) [118-122]. Activity of mature DGCs is thought to modulate survival of 1 to 2-week-old adult-born DGCs via hilar parvalbumin interneurons [181], whereas multiple lines of evidence suggest that $\sim 2$ to 4 -week-old adult-born DGCs compete with mature DGCs to receive glutamatergic perforant path inputs to integrate into the circuit $[121,128]$ (reviewed in [113]).

We asked whether we could bias competition in favor of the adult-born DGCs by decreasing the spine density of mature DGCs [118]. Using an inducible and reversible genetic system, we overexpressed a negative regulator of dendritic spines, the transcription factor Klf9 [182], in mature DGCs. Klf9 overexpression decreased mature DGC spine density, and this resulted in profound increase in survival of competing adult-born DGCs. Virally mediated deletion of the cytoskeletal factor, Rac1, in mature DGCs produced a similar increase in the number of immature adult-born DGCs. These data support previous findings that $\sim 2$ to 4-week-old adult-born DGCs (the age at which they begin to receive glutamatergic inputs) are susceptible to competition-induced cell death [121]. Furthermore, our data are consistent with a study showing that genetic enhancement or ablation of adult-born DGCs decreases or increases EPSCs in mature DGCs, respectively [161].

We harnessed our genetic system to determine the impact of enhancing adult hippocampal neurogenesis during aging on encoding and memory precision [118]. We found that middleaged and aged mice with enhanced adult hippocampal neurogenesis, unlike controls, were able to discriminate between 2 similar contexts when tested 1 day or 2 weeks after training. In addition, we assessed population-based coding in middle-aged mice. As with adult mice, expansion of the 5 to 8-week-old adult-born DGC population in middle-aged mice reduced overlap between DGC ensembles activated by similar contexts, suggesting that improved global remapping may underlie the improvements in behavioral discrimination (Fig. 1).

As stated earlier, it is unlikely that declines in adult hippocampal neurogenesis drive age-related memory decline. However, because adult hippocampal neurogenesis may promote "forgetting", or clearance of previously encoded memories [183] through neuronal competition, it is plausible that age-associated declines in adult hippocampal neurogenesis may drive cognitive inflexibility and impair resolution of memory interference.

\section{Removing the Brakes on Neural Stem Cell Activation}

Adult hippocampal neurogenesis declines with age (reviewed in [133]); however, the factors responsible for this decline are poorly understood. Humans show a less precipitous decline than rodents [111]. A number of reasons have been suggested for this decline: inability of the stem cell/progenitor populations to respond to proliferative signals (exhaustion of the stem cell niche [136], but see [184]), or reduced proliferation [185-187], lack of proneurogenic or plasticity factors [188, 189], or the presence of factors inhibiting neurogenesis - molecular 'brakes' [172, 174] (reviewed in [178]). Importantly, the aged stem cell population remains capable of increasing neurogenesis in response to exercise, neurotrophins, and systemic factors present in young blood (reviewed in [133, 178]). Further, recent work from our laboratory suggests that the aged neurogenic niche in the hippocampus is capable of responding to neurogenic stimuli and local circuit changes [118]. Overexpression of Klf9 in mature DGCs resulted in a decrease in mature DGC spines and an $\sim 2$-fold increase in survival of adult-born DGCs in adult, middle-aged, and aged mice. However, Klf9 overexpression, but not elimination of mature DGC spines alone, resulted in increased in neural stem cell activation. This increase in neural stem cell activation was significantly greater in middle-aged and aged mice than adult mice, suggesting that Klf9 overexpression in mature DGCs 
may non-cell autonomously relieve a molecular "brake" that restrains proliferation in aged animals.

Multiple candidates for these age-related brakes have been suggested. The technique of heterochronic parabiosis entails surgically linking the circulatory systems of 2 animals of different ages, and has enabled identification of systemic factors present in aged blood that inhibit neurogenesis in young animals $[172,174]$. In addition, the increases in microglial activation or corticosteroid levels in aged animals might negatively impact hippocampal neurogenesis. Microglia respond to inflammation [190], microglial levels correlate with the extent of neurogenesis in aged animals [191], and inhibition of microglia increased neurogenesis [192, 193], although the picture is still incomplete [194]. Further, corticosteroid levels ([195], but see [196]), and corticosteroid receptor expression on precursor cells [197] increase in older age and prevention of glucocorticoid-mediated effects maintains higher neurogenesis levels in aging rats [195, 198, 199].

\section{Towards Healthy Cognitive Aging in the Future}

Stimulation of adult hippocampal neurogenesis holds potential for optimizing DG-CA3 functions to maintain memory precision in aging, but major challenges remain (Fig. 3). Although our work suggests that increasing the population of adult-born DGCs in middle-aged and aged mice restores memory precision and improves global remapping, the question remains as to whether this strategy is applicable in humans. Modulating competition between mature DGCs and integrating DGCs provides selective, reversible control over adult-born DGC survival, but strategies to accomplish this in humans are currently lacking. However, activation of the perforant pathway has been shown to increase the survival of immature adult-born DGCs in mice [200], which may have functionally similar consequences on memory precision. As EC to DG connectivity is impaired with aging [96], such stimulation in aged individuals may have beneficial effects. While preliminary studies employing EC deep brain stimulation in humans resulted in improved memory performance [201], larger-scale studies have found memory impairments [202]. It is likely that further analysis and understanding of circuitry is required for successful implementation of EC stimulation protocols to improve memory in adulthood and aging.

Currently, several strategies identified in mice to promote neural stem cell activation or enhance neuronal survival might be applicable to humans. The Food and Drug Administration-



Fig. 3 Schema for how rodent studies edify novel therapeutic neurogenesis-based strategies to optimize dentate gyrus (DG)-CA3 circuit functions and improve memory in aged humans. Deficits in pattern separation/completion balance in DG-CA3 are observed/ inferred in both rodents and humans, albeit at different levels of imaging resolution. Rodent studies permit elucidation of circuit-based mechanisms by which neurogenesis modulates DG-CA3 functions. Identification of circuit-based mechanisms facilitates development of strategies that target these mechanisms to promote DG-CA3 functions. Development of novel imaging approaches to visualize adult hippocampal neurogenesis or capture pattern separation at higher resolution will permit evaluation of efficacy of new therapeutic strategies. fMRI = functional magnetic resonance imaging; PET = positron emission tomography; NMR = nuclear magnetic resonance; $\mathrm{EC}=$ entorhinal cortex 
approved diabetes drug metformin was shown to increase neurogenesis in mice [203, 204], as was the NMDA antagonist memantine [205], which has also shown efficacy in clinical trials for Alzheimer's disease (reviewed in [206]). Likewise, development of some small-molecule compounds that increase neurogenesis in mice may prove to have the same effect in humans [207]. Inhibition of inflammation or corticosteroids may mitigate some age-related decline in neurogenesis in humans as in rodents (reviewed in [208, 209]). In addition, exercise promotes neurogenesis in mice and may have similar effects in humans ([210]; reviewed in [211, 212]). Exercise has beneficial effects on cognition in aged humans [213, 214], although whether increased neurogenesis mediates the improvements is unknown (reviewed in [215]). The beneficial effects of exercise may be transmitted via exercise-induced, musclederived factors $[216,217]$ that act via promotion of neurogenesis [217].

Validation of these strategies to promote neurogenesis in humans will require the ability to visualize adult hippocampal neurogenesis in humans. This would allow efficient testing for correlations between behavioral task performance and levels of neurogenesis, with the goal of determining if increasing neurogenesis in humans improves memory performance in humans as it does in mice. Attempts have been made to image neural stem cells in vivo using MRI [218], but these remain subject to confirmation [219-221]. In addition, another study found measures of cerebral blood volume in mice correlated with increases in neurogenesis following exercise, and found similar increases in cerebral blood volume in exercising humans [222]. Another avenue is development of positron emission tomography ligands or tracers that would afford detection of neural stem cells or adult-born DGCs (Fig. 3) [223].

Increased understanding of how the connectivity of the DG-CA3 circuit causally relates to its function will inspire development of connectivity-based cognitive enhancers. As adult-born DGCs may contribute to DG-CA3 function through recruitment of inhibition, strategies to re-engineer connectivity between mature DGCs and stratum lucidum interneurons or directly promote inhibition onto CA3 neurons may bypass the need to stimulate neurogenesis ([147] and $\mathrm{N}$. Guo and A. Sahay, unpublished observations). A note of caution: forced expression of molecules in developing circuits to rewire them may impair their functions. Overexpressing neuroligin-2 in adult-born DGCs increased their survival and synaptic contacts, but impaired memory performance [119]. Ultra-high-resolution fMRI at $7 \mathrm{~T}$ and multivariate pattern analysis will permit high-resolution assessment of how DGCA3 circuit-based strategies described above impact pattern separation and completion in aged individuals (Fig. 3) [53].

Analysis of DG-CA3 connectivity, and age-related changes at the cellular level, would be facilitated by the development of empirical human models. The generation of human hippocampal neurons through direct reprogramming of fibroblasts, or directed differentiation of induced pluripotent stem cells, holds promise to delineate the neurobiological changes in aging at a cellular level [224, 225]. Although protocols have been pioneered to generate DGCs and inhibitory interneurons [226-228], we know much less about the ontogeny and process to generate CA3 pyramidal neurons. Ultimately, the generation of these different cell-types de novo or within organoids [229] will catalyze generation of human DG-CA3 circuits that permit high-resolution structure-function studies and facilitate identification of strategies to modulate connectivity at aged human DG-CA3 synapses. Further, these strategies will allow assessment of the contribution of human DGCs with different age-specific properties to excitation-inhibition balance. A detailed understanding of circuit mechanisms by which adult born DGCs contribute to encoding functions in adulthood and aging will ultimately edify new therapeutic strategies to combat age-related memory impairments.

Acknowledgments We thank members of the Sahay laboratory for their comments on the manuscript. A.S acknowledges grant support from US National Institutes of Health Biobehavioral Research Awards for Innovative New Scientists (BRAINS) 1-R01MH104175, NIH-NIA 1R01AG048908-01A1, 2017 NARSAD Independent Investigator Award, Ellison Medical Foundation New Scholar in Aging, Whitehall Foundation, Inscopix Decode award, Ellison Family Philanthropic support, and Harvard Neurodiscovery Center/MADRC Center Pilot Grant Award.

\section{References}

1. Hedden T, Gabrieli JD. Insights into the ageing mind: a view from cognitive neuroscience. Nat Rev Neurosci 2004;5(2):87-96.

2. Salthouse TA. Shared and unique influences on age-related cognitive change. Neuropsychology 2017;31(1):11-19.

3. Drag LL, Bieliauskas LA. Contemporary review 2009: cognitive aging. J Geriatr Psychiatry Neurol 2010;23(2):75-93.

4. Blazer DG, Wallace RB. Cognitive aging: what every geriatric psychiatrist should know. Am J Geriatr Psychiatry 2016;24(9): 776-781.

5. Centers for Disease Control and Prevention (CDC). Self-reported increased confusion or memory loss and associated functional difficulties among adults aged $\geq 60$ years - 21 States, 2011 . MMWR Morb Mortal Wkly Rep 2013;62(18):347-350.

6. Yaffe K, Fiocco AJ, Lindquist K, et al. Predictors of maintaining cognitive function in older adults: the Health $\mathrm{ABC}$ study. Neurology 2009;72(23):2029-2035.

7. Holsinger T, Deveau J, Boustani M, Williams JW, Jr. Does this patient have dementia? JAMA 2007;297(21):2391-2404.

8. Moyer VA. Screening for cognitive impairment in older adults: U.S. Preventive Services Task Force recommendation statement. Ann Intern Med 2014;160(11):791-797.

9. Erickson CA, Barnes CA. The neurobiology of memory changes in normal aging. Exp Gerontol 2003;38(1-2):61-69.

10. Stephan BC, Matthews FE, McKeith IG, et al. Early cognitive change in the general population: how do different definitions work? J Am Geriatr Soc 2007;55(10):1534-1540. 
11. Gallagher M, Colantuoni C, Eichenbaum H, et al. Individual differences in neurocognitive aging of the medial temporal lobe. Age (Dordr) 2006;28(3):221-233.

12. Sperling R. Functional MRI studies of associative encoding in normal aging, mild cognitive impairment, and Alzheimer's disease. Ann N Y Acad Sci 2007;1097:146-155.

13. Burke SN, Ryan L, Barnes CA. Characterizing cognitive aging of recognition memory and related processes in animal models and in humans. Front Aging Neurosci 2012;4:15.

14. Leal SL, Yassa MA. Neurocognitive aging and the hippocampus across species. Trends Neurosci 2015;38(12):800-812.

15. Holden HM, Gilbert PE. Less efficient pattern separation may contribute to age-related spatial memory deficits. Front Aging Neurosci 2012;4:9.

16. Rapp PR, Rosenberg RA, Gallagher M. An evaluation of spatial information processing in aged rats. Behav Neurosci 1987;101(1): 3-12.

17. Gallagher M, Pelleymounter MA. Spatial learning deficits in old rats: a model for memory decline in the aged. Neurobiol Aging 1988;9(5-6):549-556.

18. Gage FH, Dunnett SB, Bjorklund A. Age-related impairments in spatial memory are independent of those in sensorimotor skills. Neurobiol Aging 1989;10(4):347-352.

19. Uttl B, Graf P. Episodic spatial memory in adulthood. Psychol Aging 1993;8(2):257-273.

20. Parkin AJ, Ward J, Squires EJ, Furbear H, Clark A, Townshend J. Data-driven recognition memory: a new technique and some data on age differences. Psychonom Bull Rev 2001;8(4):812-819.

21. Henkel LA, Johnson MK, De Leonardis DM. Aging and source monitoring: cognitive processes and neuropsychological correlates. J Exp Psychol Gen 1998;127(3):251-268.

22. Friedman D. Event-related brain potential investigations of memory and aging. Biol Psychol 2000;54(1-3):175-206.

23. Toner CK, Pirogovsky E, Kirwan CB, Gilbert PE. Visual object pattern separation deficits in nondemented older adults. Learn Mem 2009;16(5):338-342.

24. Yassa MA, Lacy JW, Stark SM, Albert MS, Gallagher M, Stark CE. Pattern separation deficits associated with increased hippocampal CA3 and dentate gyrus activity in nondemented older adults. Hippocampus 2011;21(9):968-979.

25. Yassa MA, Stark CE. Pattern separation in the hippocampus. Trends Neurosci 2011;34(10):515-525.

26. Wilson IA, Gallagher M, Eichenbaum H, Tanila H. Neurocognitive aging: prior memories hinder new hippocampal encoding. Trends Neurosci 2006;29(12):662-670.

27. O'Reilly RC, McClelland JL. Hippocampal conjunctive encoding, storage, and recall: avoiding a trade-off. Hippocampus 1994;4(6): 661-682.

28. Rolls ET, Kesner RP. A computational theory of hippocampal function, and empirical tests of the theory. Prog Neurobiol 2006;79(1):1-48.

29. Treves A, Tashiro A, Witter MP, Moser EI. What is the mammalian dentate gyrus good for? Neuroscience 2008;154(4):11551172.

30. Lee I, Yoganarasimha D, Rao G, Knierim JJ. Comparison of population coherence of place cells in hippocampal subfields CA1 and CA3. Nature 2004:430(6998):456-459.

31. Vazdarjanova A, Guzowski JF. Differences in hippocampal neuronal population responses to modifications of an environmental context: evidence for distinct, yet complementary, functions of CA3 and CA1 ensembles. J Neurosci 2004;24(29):6489-6496.

32. Leutgeb S, Leutgeb JK, Treves A, Moser MB, Moser EI. Distinct ensemble codes in hippocampal areas CA3 and CA1. Science 2004;305(5688):1295-1298.
33. Leutgeb JK, Leutgeb S, Moser MB, Moser EI. Pattern separation in the dentate gyrus and CA3 of the hippocampus. Science 2007;315(5814):961-966.

34. Guzowski JF, Knierim JJ, Moser EI. Ensemble dynamics of hippocampal regions CA3 and CA1. Neuron 2004;44(4):581-584.

35. Knierim JJ, Neunuebel JP. Tracking the flow of hippocampal computation: Pattern separation, pattern completion, and attractor dynamics. Neurobiol Learn Mem 2016;129:38-49.

36. Kesner RP, Rolls ET. A computational theory of hippocampal function, and tests of the theory: new developments. Neurosci Biobehav Rev 2015;48:92-147.

37. Gilbert PE, Kesner RP. The role of the dorsal CA3 hippocampal subregion in spatial working memory and pattern separation. Behav Brain Res 2006;169(1):142-149.

38. Gilbert PE, Kesner RP, Lee I. Dissociating hippocampal subregions: double dissociation between dentate gyrus and CA1. Hippocampus 2001;11(6):626-636.

39. McHugh TJ, Jones MW, Quinn JJ, et al. Dentate gyrus NMDA receptors mediate rapid pattern separation in the hippocampal network. Science 2007;317(5834):94-99.

40. Nakazawa K, Quirk MC, Chitwood RA, et al. Requirement for hippocampal CA3 NMDA receptors in associative memory recall. Science 2002;297(5579):211-218.

41. Marr D. Simple memory: a theory for archicortex. Philos Trans R Soc Lond B Biol Sci 1971;262(841):23-81.

42. Hunsaker MR, Kesner RP. The operation of pattern separation and pattern completion processes associated with different attributes or domains of memory. Neurosci Biobehav Rev 2013;37(1):36-58.

43. Guzman SJ, Schlogl A, Frotscher M, Jonas P. Synaptic mechanisms of pattern completion in the hippocampal CA3 network. Science 2016;353(6304): 1117-1123.

44. Neunuebel JP, Knierim JJ. CA3 retrieves coherent representations from degraded input: direct evidence for CA3 pattern completion and dentate gyrus pattern separation. Neuron 2014;81(2):416-427.

45. Deng W, Mayford M, Gage FH. Selection of distinct populations of dentate granule cells in response to inputs as a mechanism for pattern separation in mice. Elife 2013;2:e00312.

46. Neunuebel JP, Yoganarasimha D, Rao G, Knierim JJ. Conflicts between local and global spatial frameworks dissociate neural representations of the lateral and medial entorhinal cortex. $\mathrm{J}$ Neurosci 2013;33(22):9246-9258.

47. Neunuebel JP, Knierim JJ. Spatial firing correlates of physiologically distinct cell types of the rat dentate gyrus. J Neurosci 2012;32(11):3848-3858.

48. GoodSmith D, Chen X, Wang C, et al. Spatial representations of granule cells and mossy cells of the dentate gyrus. Neuron 2017;93(3):677-690.

49. Danielson NB, Turi GF, Ladow M, et al. In vivo imaging of dentate gyrus mossy cells in behaving mice. Neuron 2017;93(3):5529.

50. Senzai Y, Buzsaki G. Physiological properties and behavioral correlates of hippocampal granule cells and mossy cells. Neuron 2017;93(3):691-704

51. Bakker A, Kirwan CB, Miller M, Stark CE. Pattern separation in the human hippocampal CA3 and dentate gyrus. Science 2008;319(5870):1640-1642.

52. Lacy JW, Yassa MA, Stark SM, Muftuler LT, Stark CE. Distinct pattern separation related transfer functions in human CA3/dentate and CA1 revealed using high-resolution fMRI and variable mnemonic similarity. Learn Mem 2011;18(1):15-18.

53. Berron D, Schutze H, Maass A, et al. Strong Evidence for Pattern Separation in Human Dentate Gyrus. J Neurosci 2016;36(29): 7569-7579.

54. Kyle CT, Stokes JD, Lieberman JS, Hassan AS, Ekstrom AD. Successful retrieval of competing spatial environments in humans 
involves hippocampal pattern separation mechanisms. Elife $2015 ; 4$.

55. Baker S, Vieweg P, Gao F, et al. The human dentate gyrus plays a necessary role in discriminating new memories. Curr Biol 2016;26(19):2629-2634.

56. Gracian EI, Shelley LE, Morris AM, Gilbert PE. Age-related changes in place learning for adjacent and separate locations. Neurobiol Aging 2013;34(10):2304-2309.

57. Liu X, Ramirez S, Pang PT, et al. Optogenetic stimulation of a hippocampal engram activates fear memory recall. Nature 2012;484(7394):381-385.

58. Ramirez S, Liu X, Lin PA, et al. Creating a false memory in the hippocampus. Science 2013;341(6144):387-391.

59. O'Keefe J, Dostrovsky J. The hippocampus as a spatial map. Preliminary evidence from unit activity in the freely-moving rat. Brain Res 1971;34(1):171-175.

60. Wilson MA, McNaughton BL. Dynamics of the hippocampal ensemble code for space. Science 1993;261(5124):1055-1058.

61. Rosenzweig ES, Barnes CA. Impact of aging on hippocampal function: plasticity, network dynamics, and cognition. Prog Neurobiol 2003;69(3):143-179.

62. Barnes CA, Suster MS, Shen J, McNaughton BL. Multistability of cognitive maps in the hippocampus of old rats. Nature 1997;388(6639):272-275.

63. Rosenzweig ES, Redish AD, McNaughton BL, Barnes CA. Hippocampal map realignment and spatial learning. Nat Neurosci 2003;6(6):609-615.

64. Tanila H, Shapiro ML, Eichenbaum H. Discordance of spatial representation in ensembles of hippocampal place cells. Hippocampus 1997;7(6):613-623.

65. Tanila H, Shapiro M, Gallagher M, Eichenbaum H. Brain aging: changes in the nature of information coding by the hippocampus. $\mathrm{J}$ Neurosci 1997;17(13):5155-5166.

66. Wilson IA, Ikonen S, McMahan RW, Gallagher M, Eichenbaum $\mathrm{H}$, Tanila H. Place cell rigidity correlates with impaired spatial learning in aged rats. Neurobiol Aging 2003;24(2):297-305.

67. Wilson IA, Ikonen S, Gallagher M, Eichenbaum H, Tanila H. Ageassociated alterations of hippocampal place cells are subregion specific. J Neurosci 2005;25(29):6877-6886.

68. Wilson IA, Ikonen S, Gureviciene I, et al. Cognitive aging and the hippocampus: how old rats represent new environments. J Neurosci 2004;24(15):3870-3878.

69. Guzowski JF, McNaughton BL, Barnes CA, Worley PF. Environment-specific expression of the immediate-early gene Arc in hippocampal neuronal ensembles. Nat Neurosci 1999;2(12):1120-1124.

70. Marrone DF, Adams AA, Satvat E. Increased pattern separation in the aged fascia dentata. Neurobiol Aging 2011;32(12):2317.

71. Vieweg P, Stangl M, Howard LR, Wolbers T. Changes in pattern completion-a key mechanism to explain age-related recognition memory deficits? Cortex 2015;64:343-351.

72. Yassa MA, Mattfeld AT, Stark SM, Stark CE. Age-related memory deficits linked to circuit-specific disruptions in the hippocampus. Proc Natl Acad Sci U S A 2011;108(21):8873-8878.

73. Bakker A, Krauss GL, Albert MS, et al. Reduction of hippocampal hyperactivity improves cognition in amnestic mild cognitive impairment. Neuron 2012;74(3):467-474.

74. West MJ, Coleman PD, Flood DG, Troncoso JC. Differences in the pattern of hippocampal neuronal loss in normal ageing and Alzheimer's disease. Lancet 1994;344(8925):769-772.

75. Rapp PR, Gallagher M. Preserved neuron number in the hippocampus of aged rats with spatial learning deficits. Proc Natl Acad Sci U S A 1996;93(18):9926-9930.

76. Rasmussen T, Schliemann T, Sorensen JC, Zimmer J, West MJ. Memory impaired aged rats: no loss of principal hippocampal and subicular neurons. Neurobiol Aging 1996;17(1):143-147.
77. Keuker JI, Luiten PG, Fuchs E. Preservation of hippocampal neuron numbers in aged rhesus monkeys. Neurobiol Aging 2003;24(1):157-165.

78. Calhoun ME, Kurth D, Phinney AL, et al. Hippocampal neuron and synaptophysin-positive bouton number in aging C57BL/6 mice. Neurobiol Aging 1998;19(6):599-606.

79. De Leon MJ, George AE, Golomb J, et al. Frequency of hippocampal formation atrophy in normal aging and Alzheimer's disease. Neurobiol Aging 1997;18(1):1-11.

80. Raz N, Ghisletta P, Rodrigue KM, Kennedy KM, Lindenberger U. Trajectories of brain aging in middle-aged and older adults: regional and individual differences. Neuroimage 2010;51(2):501511.

81. Raz N, Lindenberger U, Rodrigue KM, et al. Regional brain changes in aging healthy adults: general trends, individual differences and modifiers. Cereb Cortex 2005;15(11):1676-1689.

82. Small SA, Schobel SA, Buxton RB, Witter MP, Barnes CA. A pathophysiological framework of hippocampal dysfunction in ageing and disease. Nat Rev Neurosci 2011;12(10):585-601.

83. Raz N, Gunning-Dixon FM, Head D, Dupuis JH, Acker JD. Neuroanatomical correlates of cognitive aging: evidence from structural magnetic resonance imaging. Neuropsychology 1998;12(1):95-114.

84. Rosen AC, Prull MW, Gabrieli JD, et al. Differential associations between entorhinal and hippocampal volumes and memory performance in older adults. Behav Neurosci 2003;117(6):11501160.

85. Small SA, Chawla MK, Buonocore M, Rapp PR, Barnes CA. Imaging correlates of brain function in monkeys and rats isolates a hippocampal subregion differentially vulnerable to aging. Proc Natl Acad Sci U S A 2004;101(18):7181-7186.

86. Geinisman Y, deToledo-Morrell L, Morrell F, Persina IS, Rossi M. Age-related loss of axospinous synapses formed by two afferent systems in the rat dentate gyrus as revealed by the unbiased stereological dissector technique. Hippocampus 1992;2(4):437-444.

87. Geinisman Y, de Toledo-Morrell L, Morrell F. Loss of perforated synapses in the dentate gyrus: morphological substrate of memory deficit in aged rats. Proc Natl Acad Sci U S A 1986;83(9):30273031.

88. Morrison JH, Baxter MG. The ageing cortical synapse: hallmarks and implications for cognitive decline. Nat Rev Neurosci 2012;13(4):240-250.

89. Barnes CA, McNaughton BL. Physiological compensation for loss of afferent synapses in rat hippocampal granule cells during senescence. J Physiol 1980;309:473-485.

90. Barnes CA. Memory deficits associated with senescence: a neurophysiological and behavioral study in the rat. J Comp Physiol Psychol 1979;93(1):74-104.

91. Barnes CA. Normal aging: regionally specific changes in hippocampal synaptic transmission. Trends Neurosci 1994;17(1):13-18.

92. Chawla MK, Barnes CA. Hippocampal granule cells in normal aging: insights from electrophysiological and functional imaging experiments. Prog Brain Res 2007;163:661-678.

93. Chawla MK, Guzowski JF, Ramirez-Amaya V, et al. Sparse, environmentally selective expression of Arc RNA in the upper blade of the rodent fascia dentata by brief spatial experience. Hippocampus 2005;15(5):579-586.

94. Rogalski E, Stebbins GT, Barnes CA, et al. Age-related changes in parahippocampal white matter integrity: a diffusion tensor imaging study. Neuropsychologia 2012;50(8):1759-1765.

95. Stoub TR, Barnes CA, Shah RC, Stebbins GT, Ferrari C, deToledo-Morrell L. Age-related changes in the mesial temporal lobe: the parahippocampal white matter region. Neurobiol Aging 2012;33(7):1168-1176.

96. Yassa MA, Muftuler LT, Stark CE. Ultrahigh-resolution microstructural diffusion tensor imaging reveals perforant path 
degradation in aged humans in vivo. Proc Natl Acad Sci U S A 2010;107(28):12687-12691.

97. Simkin D, Hattori S, Ybarra N, et al. Aging-related hyperexcitability in CA3 Pyramidal neurons is mediated by enhanced a-type $\mathrm{K}+$ channel function and expression. J Neurosci 2015;35(38): 13206-13218.

98. Villanueva-Castillo C, Tecuatl C, Herrera-Lopez G, Galvan EJ. Aging-related impairments of hippocampal mossy fibers synapses on CA3 pyramidal cells. Neurobiol Aging 2017;49:119-137.

99. Thome A, Gray DT, Erickson CA, Lipa P, Barnes CA. Memory impairment in aged primates is associated with region-specific network dysfunction. Mol Psychiatry 2016;21(9):1257-1262.

100. Acsady L, Kamondi A, Sik A, Freund T, Buzsaki G. GABAergic cells are the major postsynaptic targets of mossy fibers in the rat hippocampus. J Neurosci 1998;18(9):3386-3403.

101. McBain CJ. Differential mechanisms of transmission and plasticity at mossy fiber synapses. Prog Brain Res 2008;169:225-240.

102. Galvan EJ, Cosgrove KE, Barrionuevo G. Multiple forms of longterm synaptic plasticity at hippocampal mossy fiber synapses on interneurons. Neuropharmacology 2011;60(5):740-747.

103. Cadiacio CL, Milner TA, Gallagher M, Pierce JP. Hilar neuropeptide $\mathrm{Y}$ interneuron loss in the aged rat hippocampal formation. Exp Neurol 2003;183(1):147-158.

104. Stanley DP, Shetty AK. Aging in the rat hippocampus is associated with widespread reductions in the number of glutamate decarboxylase-67 positive interneurons but not interneuron degeneration. J Neurochem 2004;89(1):204-216.

105. Vela J, Gutierrez A, Vitorica J, Ruano D. Rat hippocampal GABAergic molecular markers are differentially affected by ageing. J Neurochem 2003;85(2):368-377.

106. Altman J, Das GD. Autoradiographic and histological evidence of postnatal hippocampal neurogenesis in rats. J Comp Neurol 1965;124(3):319-335.

107. Eriksson PS, Perfilieva E, Bjork-Eriksson T, et al. Neurogenesis in the adult human hippocampus. Nat Med 1998;4(11):1313-1317.

108. Boldrini M, Hen R, Underwood MD, et al. Hippocampal angiogenesis and progenitor cell proliferation are increased with antidepressant use in major depression. Biol Psychiatry 2012;72(7):562571.

109. Boldrini M, Underwood MD, Hen R, et al. Antidepressants increase neural progenitor cells in the human hippocampus. Neuropsychopharmacology 2009;34(11):2376-2389.

110. Knoth R, Singec I, Ditter M, et al. Murine features of neurogenesis in the human hippocampus across the lifespan from 0 to 100 years. PLOS ONE 2010;5(1):e8809.

111. Spalding KL, Bergmann O, Alkass K, et al. Dynamics of hippocampal neurogenesis in adult humans. Cell 2013;153(6):12191227.

112. Ming GL, Song H. Adult neurogenesis in the mammalian brain: significant answers and significant questions. Neuron 2011;70(4): 687-702.

113. Bergami M, Berninger B. A fight for survival: the challenges faced by a newborn neuron integrating in the adult hippocampus. Dev Neurobiol 2012;72(7):1016-1031.

114. Toni N, Schinder AF. Maturation and functional integration of new granule cells into the adult hippocampus. Cold Spring Harb Perspect Biol 2015;8(1):a018903.

115. Deshpande A, Bergami M, Ghanem A, et al. Retrograde monosynaptic tracing reveals the temporal evolution of inputs onto new neurons in the adult dentate gyrus and olfactory bulb. Proc Natl Acad Sci U S A 2013;110(12):E1152-E1161.

116. Bergami M, Masserdotti G, Temprana SG, et al. A critical period for experience-dependent remodeling of adult-born neuron connectivity. Neuron 2015;85(4):710-717.

117. Vivar C, Potter MC, Choi J, et al. Monosynaptic inputs to new neurons in the dentate gyrus. Nat Commun 2012;3:1107.
118. McAvoy KM, Scobie KN, Berger S, et al. Modulating neuronal competition dynamics in the dentate gyrus to rejuvenate aging memory circuits. Neuron 2016;91(6):1356-1373.

119. Krzisch M, Fulling C, Jabinet L, et al. Synaptic adhesion molecules regulate the integration of new granule neurons in the postnatal mouse hippocampus and their impact on spatial memory. Cereb Cortex 2016 Jul 29 [Epub ahead of print].

120. Sultan S, Li L, Moss J, et al. Synaptic integration of adult-born hippocampal neurons is locally controlled by astrocytes. Neuron 2015;88(5):957-972.

121. Tashiro A, Sandler VM, Toni N, Zhao C, Gage FH. NMDA-receptor-mediated, cell-specific integration of new neurons in adult dentate gyrus. Nature 2006;442(7105):929-933.

122. Doengi M, Krupp AJ, Korber N, Stein V. SynCAM 1 improves survival of adult-born neurons by accelerating synapse maturation. Hippocampus 2016;26(3):319-328.

123. Denny CA, Burghardt NS, Schachter DM, Hen R, Drew MR. 4-6 week old adult-born hippocampal neurons influence noveltyevoked exploration and contextual fear conditioning. Hippocampus 2012;22:1188-1201.

124. Ge S, Yang CH, Hsu KS, Ming GL, Song H. A critical period for enhanced synaptic plasticity in newly generated neurons of the adult brain. Neuron 2007;54(4):559-566.

125. Marin-Burgin A, Mongiat LA, Pardi MB, Schinder AF. Unique processing during a period of high excitation/inhibition balance in adult-born neurons. Science 2012;335(6073):1238-1242.

126. Gu Y, Arruda-Carvalho M, Wang J, et al. Optical controlling reveals time-dependent roles for adult-born dentate granule cells. Nat Neurosci 2012;15(12):1700-1706.

127. Toni N, Laplagne DA, Zhao C, et al. Neurons born in the adult dentate gyrus form functional synapses with target cells. Nat Neurosci 2008;11(8):901-907.

128. Toni N, Teng EM, Bushong EA, et al. Synapse formation on neurons born in the adult hippocampus. Nat Neurosci 2007;10(6):727-734

129. Schmidt-Hieber C, Jonas P, Bischofberger J. Enhanced synaptic plasticity in newly generated granule cells of the adult hippocampus. Nature 2004;429(6988):184-187.

130. Saxe MD, Battaglia F, Wang JW, et al. Ablation of hippocampal neurogenesis impairs contextual fear conditioning and synaptic plasticity in the dentate gyrus. Proc Natl Acad Sci U S A 2006;103(46):17501-17506.

131. Snyder JS, Kee N, Wojtowicz JM. Effects of adult neurogenesis on synaptic plasticity in the rat dentate gyrus. J Neurophysiol 2001;85(6):2423-2431.

132. Zhuo JM, Tseng HA, Desai M, et al. Young adult born neurons enhance hippocampal dependent performance via influences on bilateral networks. eLife 2016;5.

133. Kempermann G. Activity dependency and aging in the regulation of adult neurogenesis. Cold Spring Harb Perspect Biol 2015;7(11).

134. Ben Abdallah NM, Slomianka L, Vyssotski AL, Lipp HP. Early age-related changes in adult hippocampal neurogenesis in C57 mice. Neurobiol Aging 2010;31(1):151-161.

135. Artegiani B, Calegari F. Age-related cognitive decline: can neural stem cells help us? Aging 2012;4(3):176-186.

136. Encinas JM, Sierra A, Valcarcel-Martin R, Martin-Suarez S. A developmental perspective on adult hippocampal neurogenesis. Int J Dev Neurosci 2013;31(7):640-645.

137. Bizon JL, Gallagher M. Production of new cells in the rat dentate gyrus over the lifespan: relation to cognitive decline. Eur $\mathrm{J}$ Neurosci 2003;18(1):215-219.

138. Clelland CD, Choi M, Romberg C, et al. A functional role for adult hippocampal neurogenesis in spatial pattern separation. Science 2009;325(5937):210-213. 
139. Nakashiba T, Cushman JD, Pelkey KA, et al. Young dentate granule cells mediate pattern separation, whereas old granule cells facilitate pattern completion. Cell 2012;149(1):188-201.

140. Kheirbek MA, Klemenhagen KC, Sahay A, Hen R. Neurogenesis and generalization: a new approach to stratify and treat anxiety disorders. Nat Neurosci 2012;15(12):1613-1620.

141. Sahay A, Scobie KN, Hill AS, et al. Increasing adult hippocampal neurogenesis is sufficient to improve pattern separation. Nature 2011;472(7344):466-470.

142. Tronel S, Belnoue L, Grosjean N, et al. Adult-born neurons are necessary for extended contextual discrimination. Hippocampus 2012;22(2):292-298.

143. Wojtowicz JM, Askew ML, Winocur G. The effects of running and of inhibiting adult neurogenesis on learning and memory in rats. Eur J Neurosci 2008;27(6):1494-1502.

144. Garthe A, Behr J, Kempermann G. Adult-generated hippocampal neurons allow the flexible use of spatially precise learning strategies. PLOS ONE 2009;4(5):e5464.

145. Burghardt NS, Park EH, Hen R, Fenton AA. Adult-born hippocampal neurons promote cognitive flexibility in mice. Hippocampus 2012;22(9):1795-1808.

146. Niibori Y, Yu TS, Epp JR, Akers KG, Josselyn SA, Frankland PW. Suppression of adult neurogenesis impairs population coding of similar contexts in hippocampal CA3 region. Nat Commun 2012;3:1253.

147. Besnard A, Sahay A. Adult hippocampal neurogenesis, fear generalization, and stress. Neuropsychopharmacology 2016;41(1):24-44.

148. Kheirbek MA, Tannenholz L, Hen R. NR2B-dependent plasticity of adult-born granule cells is necessary for context discrimination. J Neurosci 2012;32(25):8696-8702.

149. Danielson NB, Kaifosh P, Zaremba JD, et al. Distinct contribution of adult-born hippocampal granule cells to context encoding. Neuron 2016;90(1):101-112.

150. Creer DJ, Romberg C, Saksida LM, van Praag H, Bussey TJ. Running enhances spatial pattern separation in mice. Proc Natl Acad Sci U S A 2010;107(5):2367-2372.

151. Sahay A, Wilson DA, Hen R. Pattern separation: a common function for new neurons in hippocampus and olfactory bulb. Neuron 2011;70(4):582-588.

152. Ikrar T, Guo N, He K, et al. Adult neurogenesis modifies excitability of the dentate gyrus. Front Neural Circuits 2013;7:204.

153. McAvoy K, Besnard A, Sahay A. Adult hippocampal neurogenesis and pattern separation in DG: a role for feedback inhibition in modulating sparseness to govern population-based coding. Front Syst Neurosci 2015;9:120.

154. Poo MM, Pignatelli M, Ryan TJ, et al. What is memory? The present state of the engram. BMC Biol 2016;14:40.

155. Drew LJ, Kheirbek MA, Luna VM, et al. Activation of local inhibitory circuits in the dentate gyrus by adult-born neurons. Hippocampus 2016;26(6):763-778.

156. Besnard A, Sahay A. Adult hippocampal neurogenesis, fear generalization, and stress. Neuropsychopharmacology 2016;41:24-44.

157. Stefanelli T, Bertollini C, Luscher C, Muller D, Mendez P. Hippocampal somatostatin interneurons control the size of neuronal memory ensembles. Neuron 2016;89(5):1074-1085.

158. Lacefield CO, Itskov V, Reardon T, Hen R, Gordon JA. Effects of adult-generated granule cells on coordinated network activity in the dentate gyrus. Hippocampus 2012;22(1):106-116.

159. Temprana SG, Mongiat LA, Yang SM, et al. Delayed coupling to feedback inhibition during a critical period for the integration of adult-born granule cells. Neuron 2015;85(1):116-130.

160. Restivo L, Niibori Y, Mercaldo V, Josselyn SA, Frankland PW. Development of adult-generated cell connectivity with excitatory and inhibitory cell populations in the hippocampus. J Neurosci 2015;35(29):10600-10612.

161. Adlaf EW, Vaden RJ, Niver AJ, et al. Adult-born neurons modify excitatory synaptic transmission to existing neurons. Elife 2017;6.

162. Park EH, Burghardt NS, Dvorak D, Hen R, Fenton AA. Experience-dependent regulation of dentate gyrus excitability by adult-born granule cells. J Neurosci 2015;35(33):11656-11666.

163. Ruediger S, Spirig D, Donato F, Caroni P. Goal-oriented searching mediated by ventral hippocampus early in trial-and-error learning. Nat Neurosci 2012;15(11):1563-1571.

164. Ruediger S, Vittori C, Bednarek E, et al. Learning-related feedforward inhibitory connectivity growth required for memory precision. Nature 2011;473:514-518.

165. Denny CA, Kheirbek MA, Alba EL, et al. Hippocampal memory traces are differentially modulated by experience, time, and adult neurogenesis. Neuron 2014;83(1):189-201.

166. Deng W, Saxe MD, Gallina IS, Gage FH. Adult-born hippocampal dentate granule cells undergoing maturation modulate learning and memory in the brain. J Neurosci 2009;29(43):13532-13542.

167. Snyder JS, Hong NS, McDonald RJ, Wojtowicz JM. A role for adult neurogenesis in spatial long-term memory. Neuroscience 2005;130(4):843-852.

168. Goodman T, Trouche S, Massou I, et al. Young hippocampal neurons are critical for recent and remote spatial memory in adult mice. Neuroscience 2010;171(3):769-778.

169. Pan YW, Storm DR, Xia Z. The maintenance of established remote contextual fear memory requires ERK5 MAP kinase and ongoing adult neurogenesis in the hippocampus. PLOS ONE 2012;7(11):e50455.

170. Wang W, Pan YW, Zou J, et al. Genetic activation of ERK5 MAP kinase enhances adult neurogenesis and extends hippocampusdependent long-term memory. J Neurosci 2014;34(6):2130-2147.

171. Seib DR, Corsini NS, Ellwanger K, et al. Loss of Dickkopf-1 restores neurogenesis in old age and counteracts cognitive decline. Cell Stem Cell 2013;12(2):204-214.

172. Smith LK, He Y, Park JS, et al. beta2-microglobulin is a systemic pro-aging factor that impairs cognitive function and neurogenesis. Nat Med 2015;21(8):932-937.

173. Gibbons TE, Pence BD, Petr G, et al. Voluntary wheel running, but not a diet containing (-)-epigallocatechin-3-gallate and betaalanine, improves learning, memory and hippocampal neurogenesis in aged mice. Behav Brain Res 2014;272:131-140.

174. Villeda SA, Luo J, Mosher KI, et al. The ageing systemic milieu negatively regulates neurogenesis and cognitive function. Nature 2011;477(7362):90-94.

175. van Praag H, Shubert T, Zhao C, Gage FH. Exercise enhances learning and hippocampal neurogenesis in aged mice. J Neurosci 2005;25(38):8680-8685.

176. Wu MV, Luna VM, Hen R. Running rescues a fear-based contextual discrimination deficit in aged mice. Front Syst Neurosci 2015;9:114.

177. Pardo J, Uriarte M, Console GM, et al. Insulin-like growth factor-I gene therapy increases hippocampal neurogenesis, astrocyte branching and improves spatial memory in female aging rats. Eur J Neurosci 2016;44(4):2120-2128.

178. Bouchard J, Villeda SA. Aging and brain rejuvenation as systemic events. J Neurochem 2015;132(1):5-19.

179. Drapeau E, Mayo W, Aurousseau C, Le Moal M, Piazza PV, Abrous DN. Spatial memory performances of aged rats in the water maze predict levels of hippocampal neurogenesis. Proc Natl Acad Sci U S A 2003;100(24):14385-14390.

180. Merrill DA, Karim R, Darraq M, Chiba AA, Tuszynski MH. Hippocampal cell genesis does not correlate with spatial learning ability in aged rats. J Comp Neurol 2003;459(2):201-207. 
181. Alvarez DD, Giacomini D, Yang SM, et al. A disynaptic feedback network activated by experience promotes the integration of new granule cells. Science 2016;354(6311):459-465.

182. Scobie KN, Hall BJ, Wilke SA, et al. Kruppel-like factor 9 is necessary for late-phase neuronal maturation in the developing dentate gyrus and during adult hippocampal neurogenesis. J Neurosci 2009;29(31):9875-9887.

183. Akers KG, Martinez-Canabal A, Restivo L, et al. Hippocampal neurogenesis regulates forgetting during adulthood and infancy. Science 2014;344(6184):598-602.

184. Bonaguidi MA, Wheeler MA, Shapiro JS, et al. In vivo clonal analysis reveals self-renewing and multipotent adult neural stem cell characteristics. Cell 2011;145(7):1142-1155.

185. Yang TT, Lo CP, Tsai PS, et al. Aging and exercise affect hippocampal neurogenesis via different mechanisms. PLOS ONE 2015;10(7):e0132152.

186. Lugert S, Basak O, Knuckles P, et al. Quiescent and active hippocampal neural stem cells with distinct morphologies respond selectively to physiological and pathological stimuli and aging. Cell Stem Cell 2010;6(5):445-456.

187. Rao MS, Hattiangady B, Shetty AK. The window and mechanisms of major age-related decline in the production of new neurons within the dentate gyrus of the hippocampus. Aging Cell 2006;5(6):545-558.

188. Katsimpardi L, Litterman NK, Schein PA, et al. Vascular and neurogenic rejuvenation of the aging mouse brain by young systemic factors. Science 2014;344(6184):630-634.

189. Villeda SA, Plambeck KE, Middeldorp J, et al. Young blood reverses age-related impairments in cognitive function and synaptic plasticity in mice. Nat Med 2014;20(6):659-663.

190. Cornejo F, von Bernhardi R. Age-Dependent changes in the activation and regulation of microglia. Adv Exp Med Biol 2016;949: 205-226.

191. Gebara E, Sultan S, Kocher-Braissant J, Toni N. Adult hippocampal neurogenesis inversely correlates with microglia in conditions of voluntary running and aging. Front Neurosci 2013;7:145.

192. Monje ML, Toda H, Palmer TD. Inflammatory blockade restores adult hippocampal neurogenesis. Science 2003;302(5651):17601765.

193. Kohman RA, Bhattacharya TK, Kilby C, Bucko P, Rhodes JS. Effects of minocycline on spatial learning, hippocampal neurogenesis and microglia in aged and adult mice. Behav Brain Res 2013;242:17-24.

194. Su P, Zhang J, Zhao F, Aschner M, Chen J, Luo W. The interaction between microglia and neural stem/precursor cells. Brain Res Bull 2014;109:32-38.

195. Cameron HA, McKay RD. Restoring production of hippocampal neurons in old age. Nat Neurosci 1999;2(10):894-897.

196. Heine VM, Maslam S, Joels M, Lucassen PJ. Prominent decline of newborn cell proliferation, differentiation, and apoptosis in the aging dentate gyrus, in absence of an age-related hypothalamuspituitary-adrenal axis activation. Neurobiol Aging 2004;25(3): 361-375.

197. Garcia A, Steiner B, Kronenberg G, Bick-Sander A, Kempermann G. Age-dependent expression of glucocorticoid- and mineralocorticoid receptors on neural precursor cell populations in the adult murine hippocampus. Aging Cell 2004;3(6):363-371.

198. Mayer JL, Klumpers L, Maslam S, de Kloet ER, Joels M, Lucassen PJ. Brief treatment with the glucocorticoid receptor antagonist mifepristone normalises the corticosterone-induced reduction of adult hippocampal neurogenesis. J Neuroendocrinol 2006;18(8):629-631.

199. Montaron MF, Drapeau E, Dupret D, et al. Lifelong corticosterone level determines age-related decline in neurogenesis and memory. Neurobiol Aging 2006;27(4):645-654.
200. Stone SS, Teixeira CM, Devito LM, et al. Stimulation of entorhinal cortex promotes adult neurogenesis and facilitates spatial memory. J Neurosci 2011;31(38):13469-13484.

201. Suthana N, Haneef Z, Stern J, et al. Memory enhancement and deep-brain stimulation of the entorhinal area. N Engl J Med 2012;366(6):502-510

202. Jacobs J, Miller J, Lee SA, et al. Direct electrical stimulation of the human entorhinal region and hippocampus impairs memory. Neuron 2016;92(5):983-990.

203. Wang J, Gallagher D, DeVito LM, et al. Metformin activates an atypical PKC-CBP pathway to promote neurogenesis and enhance spatial memory formation. Cell Stem Cell 2012;11(1):23-35.

204. Fatt M, Hsu K, He L, et al. Metformin acts on two different molecular pathways to enhance adult neural precursor proliferation/ self-renewal and differentiation. Stem Cell Rep 2015;5(6):988995.

205. Ishikawa R, Kim R, Namba T, Kohsaka S, Uchino S, Kida S. Time-dependent enhancement of hippocampus-dependent memory after treatment with memantine: Implications for enhanced hippocampal adult neurogenesis. Hippocampus 2014;24(7):784-793.

206. Koch HJ, Uyanik G, Fischer-Barnicol D. Memantine: a therapeutic approach in treating Alzheimer's and vascular dementia. Curr Drug Targets CNS Neurol Disord 2005;4(5):499-506.

207. Pieper AA, Xie S, Capota E, et al. Discovery of a proneurogenic neuroprotective chemical. Cell 2010;142(1):39-51.

208. de Miranda AS, Zhang CJ, Katsumoto A, Teixeira AL. Hippocampal adult neurogenesis: Does the immune system matter? J Neurol Sci 2017;372:482-495.

209. De Nicola AF, Pietranera L, Beauquis J, Ferrini MG, Saravia FE. Steroid protection in aging and age-associated diseases. Exp Gerontol 2009;44(1-2):34-40.

210. Kronenberg G, Bick-Sander A, Bunk E, Wolf C, Ehninger D, Kempermann G. Physical exercise prevents age-related decline in precursor cell activity in the mouse dentate gyrus. Neurobiol Aging 2006;27(10):1505-1513.

211. Vivar C, Potter MC, van Praag H. All about running: synaptic plasticity, growth factors and adult hippocampal neurogenesis. Curr Top Behav Neurosci 2013;15:189-210.

212. Voss MW, Vivar C, Kramer AF, van Praag H. Bridging animal and human models of exercise-induced brain plasticity. Trends Cogn Sci 2013;17(10):525-544.

213. Larson EB, Wang L, Bowen JD, et al. Exercise is associated with reduced risk for incident dementia among persons 65 years of age and older. Ann Intern Med 2006;144(2):73-81.

214. Lautenschlager NT, Cox KL, Flicker L, et al. Effect of physical activity on cognitive function in older adults at risk for Alzheimer disease: a randomized trial. JAMA 2008;300(9):1027-1037.

215. Yau SY, Gil-Mohapel J, Christie BR, So KF. Physical exerciseinduced adult neurogenesis: a good strategy to prevent cognitive decline in neurodegenerative diseases? Biomed Res Int 2014;2014:403120.

216. Agudelo LZ, Femenia T, Orhan F, et al. Skeletal muscle PGC1alpha1 modulates kynurenine metabolism and mediates resilience to stress-induced depression. Cell 2014;159(1):33-45.

217. Moon HY, Becke A, Berron D, et al. Running-induced systemic cathepsin B secretion is associated with memory function. Cell Metab 2016;24(2):332-340.

218. Manganas LN, Zhang X, Li Y, et al. Magnetic resonance spectroscopy identifies neural progenitor cells in the live human brain. Science 2007;318(5852):980-985.

219. Friedman SD. Comment on "Magnetic resonance spectroscopy identifies neural progenitor cells in the live human brain". Science 2008;321(5889):640.

220. Hoch JC, Maciejewski MW, Gryk MR. Comment on "magnetic resonance spectroscopy identifies neural progenitor cells in the live human brain". Science 2008;321(5889):640. 
221. Jansen JF, Gearhart JD, Bulte JW. Comment on "Magnetic resonance spectroscopy identifies neural progenitor cells in the live human brain". Science 2008;321(5889):640.

222. Pereira AC, Huddleston DE, Brickman AM, et al. An in vivo correlate of exercise-induced neurogenesis in the adult dentate gyrus. Proc Natl Acad Sci U S A 2007;104(13):5638-5643.

223. Ho NF, Hooker JM, Sahay A, Holt DJ, Roffman JL. In vivo imaging of adult human hippocampal neurogenesis: progress, pitfalls and promise. Mol Psychiatry 2013;18(4):404-416.

224. Mertens J, Paquola AC, Ku M, et al. Directly reprogrammed human neurons retain aging-associated transcriptomic signatures and reveal age-related nucleocytoplasmic defects. Cell Stem Cell 2015;17(6):705-718.
225. Huh CJ, Zhang B, Victor MB, et al. Maintenance of age in human neurons generated by microRNA-based neuronal conversion of fibroblasts. Elife 2016;5.

226. Colasante G, Lignani G, Rubio A, et al. Rapid conversion of fibroblasts into functional forebrain GABAergic interneurons by direct genetic reprogramming. Cell Stem Cell 2015;17(6):719734.

227. Sun AX, Yuan Q, Tan S, et al. Direct induction and functional maturation of forebrain GABAergic neurons from human pluripotent stem cells. Cell Rep 2016;16(7):1942-1953.

228. Yu DX, Di Giorgio FP, Yao J, et al. Modeling hippocampal neurogenesis using human pluripotent stem cells. Stem Cell Rep 2014;2(3):295-310.

229. Clevers H. Modeling development and disease with organoids. Cell 2016;165(7):1586-1597. 